\title{
Differential operator generalized by fractional derivatives
}

\author{
Rabha W. Ibrahim and M. Darus
}




\title{
DIFFERENTIAL OPERATOR GENERALIZED BY FRACTIONAL DERIVATIVES
}

\author{
RABHA W. IBRAHIM AND M. DARUS
}

Received March 30, 2010

\begin{abstract}
By using the fractional derivative operator of Owa and Srivastava, we define a new linear multiplier fractional differential operator. Some generalized classes of analytic functions containing this multiplier are introduced. Basic properties of these classes are studied, such as inclusion relations and coefficient bounds. Some well known subclasses are pointed out as new special cases of our results. Moreover, the Cesáro partial sums $\sigma_{m}$ of functions $f$ are considered, and sharp lower bounds for the ratios of real part of $f$ and $\sigma_{m}$ (and also of $f^{\prime}$ and $\sigma_{m}^{\prime}$ ) are determined in the unit open disk.
\end{abstract}

2000 Mathematics Subject Classification: $30 \mathrm{C} 45$

Keywords: starlike function, convex function, close to convex function, fractional derivative, inclusion relations, subordination, superordination, Cesáro partial sums

\section{INTRODUCTION}

Let $\mathscr{H}$ be the class of analytic functions in $U:=\{z \in \mathbb{C}:|z|<1\}$ and $\mathscr{H}[a, n]$ be the subclass of $\mathscr{H}$ consisting of functions of the form $f(z)=a+a_{n} z^{n}+a_{n+1} z^{n+1}+$ .... Let $\mathcal{A}$ be the class of functions of the form

$$
f(z)=z+\sum_{n=2}^{\infty} a_{n} z^{n},
$$

which are analytic in the unit disk $U$

Given two functions $f, g \in \mathcal{A}, f(z)=z+\sum_{n=2}^{\infty} a_{n} z^{n}$ and $g(z)=z+\sum_{n=2}^{\infty} b_{n} z^{n}$ their convolution or Hadamard product $f(z) * g(z)$ is defined by

$$
f(z) * g(z)=z+\sum_{n=2}^{\infty} a_{n} b_{n} z^{n},(z \in U) .
$$

The work here is fully supported by MOHE: UKM-ST-06-FRGS0244-2010. 
For several functions $f_{1}(z), \ldots, f_{m}(z) \in \mathcal{A}$,

$$
f_{1}(z) * \ldots * f_{m}(z)=z+\sum_{n=2}^{\infty}\left(a_{1 n} \ldots a_{m n}\right) z^{n}, \quad(z \in U) .
$$

A function $f \in \mathcal{A}$ is called starlike of order $\mu$ if it satisfies the following inequality

$$
\Re\left\{\frac{z f^{\prime}(z)}{f(z)}\right\}>\mu,(z \in U)
$$

for some $0 \leq \mu<1$. We denoted this by by class $\&(\mu)$. A function $f \in \mathcal{A}$ is called convex of order $\mu$ if it satisfies the following inequality

$$
\Re\left\{\frac{z f^{\prime \prime}(z)}{f^{\prime}(z)}+1\right\}>\mu,(z \in U)
$$

for some $0 \leq \mu<1$. We denoted this class $\mathcal{C}(\mu)$. Note that $f \in \mathcal{C}(\mu)$ if and only if $z f^{\prime} \in \delta(\mu)$. The following definitions are required in our present investigation.

Definition 1. (see [8] ) (Subordination Principal). For two functions $f$ and $g$ analytic in $U$, we say that the function $f(z)$ is subordinated to $g(z)$ in $U$ and write $f(z) \prec g(z)(z \in U)$, if there exists a Schwarz function $w(z)$ analytic in $U$ with $w(0)=0$, and $|w(z)|<1$, such that $f(z)=g(w(z)), z \in U$. In particular, if the function $g(z)$ is univalent in $U$, the above subordination is equivalent to $f(0)=g(0)$ and $f(U) \subset g(U)$.

Definition 2. (see [9]) (Differential subordination ) Let $\phi: \mathbb{C}^{2} \rightarrow \mathbb{C}$ and let $h$ be univalent in $U$. If $p$ is analytic in $U$ and satisfies the differential subordination $\left.\phi(p(z)), z p^{\prime}(z)\right) \prec h(z)$ then $p$ is called a solution of the differential subordination. The univalent function $q$ is called a dominant of the solutions of the differential subordination, $p \prec q$. If $p$ and $\left.\phi(p(z)), z p^{\prime}(z)\right)$ are univalent in $U$ and satisfy the differential superordination $\left.h(z) \prec \phi(p(z)), z p^{\prime}(z)\right)$, then $p$ is called a solution of the differential superordination. An analytic function $q$ is called subordinant of the solution of the differential superordination if $q \prec p$.

Definition 3. (see [10]) The fractional derivative of order $\alpha$ is defined, for a function $f(z)$, by

$$
D_{z}^{\alpha} f(z):=\frac{1}{\Gamma(1-\alpha)} \frac{d}{d z} \int_{0}^{z} \frac{f(\zeta)}{(z-\zeta)^{\alpha}} d \zeta ; 0 \leq \alpha<1,
$$

where the function $f(z)$ is analytic in simply-connected region of the complex zplane $\mathbb{C}$ containing the origin and the multiplicity of $(z-\zeta)^{-\alpha}$ is removed by requiring $\log (z-\zeta)$ to be real when $(z-\zeta)>0$.

Definition 4. [15] A function $f \in S$ (the class of univalent functions in $U$ ) is said to be in the class $S H(\mu)$ if it satisfies

$$
\left|\frac{z f^{\prime}(z)}{f(z)}-2 \mu(\sqrt{2}-1)\right|<\Re\left\{\sqrt{2} \frac{z f^{\prime}(z)}{f(z)}\right\}+2 \mu(\sqrt{2}-1)
$$


for some $\mu>0$ and for all $z \in U$.

Remark 1. Geometric interpretation: Let $\Omega(\mu):=\left\{\frac{z f^{\prime}(z)}{f(z)}, f \in H S(\mu)\right\}$ then

$$
\Omega(\mu)=\left\{\omega=u+i v: v^{2}<4 \mu u+u^{2} ; u>0\right\} .
$$

Note that $\Omega(\mu)$ is the interior of a hyperbola in the right half-plane which is symmetric with respect to the real axis and has vertex at the origin.

Define the function $\varphi_{\alpha}(a, c ; z)$ by

$$
\varphi(a, c ; z):=\sum_{n=0}^{\infty} \frac{(a)_{n}}{(c)_{n}} z^{n},(z \in U ; a \in \mathbb{R}, c \in \mathbb{R} \backslash\{0,-1,-2, \ldots\}),
$$

where $(a)_{n}$ is the Pochhammer symbol defined by

$$
(a)_{n}:=\frac{\Gamma(a+n)}{\Gamma(a)}=\left\{\begin{array}{l}
1, \\
a(a+1)(a+2) \ldots(a+n-1), \quad(n \in \mathbb{N}) .
\end{array}\right.
$$

Corresponding to the function $\varphi_{\alpha}(a, c ; z)$, Carlson and Shaffer [5] introduced a linear operator $L(a, c)$ by

$$
L(a, c) f(z):=\varphi(a, c ; z) * f(z), f \in \mathcal{A} .
$$

Note that $L(a, a)$ is the identity operator.

In [11], Owa and Srivastava introduced the operator $\Phi^{\alpha}: \mathcal{A} \rightarrow \mathcal{A}$, that is known as an extension of fractional derivative and fractional integral, as follows

$$
\begin{aligned}
\Phi^{\alpha} f(z):= & \Gamma(2-\alpha) z^{\alpha} D_{z}^{\alpha} f(z) \\
& =z+\sum_{n=2}^{\infty} \frac{\Gamma(n+1) \Gamma(2-\alpha)}{\Gamma(n+1-\alpha)} a_{n} z^{n} \\
& =\varphi(2,2-\alpha ; z) * f(z) \\
& =L(2,2-\alpha ; z) f(z) .
\end{aligned}
$$

Note that $\Phi^{0} f(z)=f(z)$. We define the linear multiplier fractional differential operator $D_{\beta, \lambda}^{k, \alpha}$ as follows

$$
\begin{gathered}
D^{0} f(z)=f(z) \\
=z+\sum_{n=2}^{\infty} a_{n} z^{n}, \\
D_{\beta, \lambda}^{1, \alpha} f(z)=(\beta-\lambda) \Phi^{\alpha} f(z)+\lambda z\left(\Phi^{\alpha} f(z)\right)^{\prime}+(1-\beta) z \\
=z+\sum_{n=2}^{\infty}\left[\frac{\Gamma(n+1) \Gamma(2-\alpha)}{\Gamma(n+1-\alpha)}\right][\lambda(n-1)+\beta] a_{n} z^{n} \\
=D_{\beta, \lambda}^{\alpha}(f(z)),
\end{gathered}
$$




$$
\begin{aligned}
& D_{\beta, \lambda}^{2, \alpha} f(z)=D_{\beta, \lambda}^{\alpha}\left(D_{\beta, \lambda}^{1, \alpha} f(z)\right) \\
&=z+\sum_{n=2}^{\infty}\left\{\left[\frac{\Gamma(n+1) \Gamma(2-\alpha)}{\Gamma(n+1-\alpha)}\right][\lambda(n-1)+\beta]\right\}^{2} a_{n} z^{n}, \\
& \vdots \\
& D_{\beta, \lambda}^{k, \alpha} f(z)=D_{\beta, \lambda}^{\alpha}\left(D_{\beta, \lambda}^{k-1, \alpha} f(z)\right) \\
&=z+\sum_{n=2}^{\infty}\left\{\left[\frac{\Gamma(n+1) \Gamma(2-\alpha)}{\Gamma(n+1-\alpha)}\right][\lambda(n-1)+\beta]\right\}^{k} a_{n} z^{n} \\
&:=z+\sum_{n=2}^{\infty} \Psi_{n, k}(\alpha, \beta, \lambda) a_{n} z^{n},
\end{aligned}
$$

for $0 \leq \alpha<1, \beta \geq 1, \lambda \geq 0$ and $k \in \mathbb{N}_{0}=\mathbb{N} \cup\{0\}$ with $D_{\beta, \lambda}^{k, \alpha} f(0)=0$.

Remark 2. (1) When $\alpha=0, \beta=1$, we have Al-Oboudi's differential operator (see [3]).

(2) When $\alpha=0, \beta=1$ and $\lambda=1$, we get Sălăgean's differential operator (see [16]).

(3) When $k=1, \beta=1$ and $\lambda=0$, we obtain the Owa-Srivastava fractional differential operator (see [11]).

(4) When $\beta=1$, we get the linear multiplier fractional differential operator $D_{\lambda}^{k, \alpha}$ that introduced in [4].

Using the operator $D_{\beta, \lambda}^{k, \alpha}$, we define the following classes.

Definition 5. Let $S H_{k, \alpha, \beta, \lambda}(\mu), k \in \mathbb{N}$ be the class of all functions $f \in \mathcal{A}$ and univalent in $U$ satisfying

$$
\left|\frac{D_{\beta, \lambda}^{k+1, \alpha} f(z)}{D_{\beta, \lambda}^{k, \alpha} f(z)}-2 \mu(\sqrt{2}-1)\right|<\Re\left\{\sqrt{2} \frac{D_{\beta, \lambda}^{k+1, \alpha} f(z)}{D_{\beta, \lambda}^{k, \alpha} f(z)}\right\}+2 \mu(\sqrt{2}-1)
$$

for some $\mu,(\mu>0)$ and for all $z \in U$.

Remark 3. Geometric interpretation: If we denote with $p_{\mu}$ the analytic and univalent functions with the properties $p_{\mu}(0)=1, p_{\mu}^{\prime}(0)>0$ and $p_{\mu}(U)=\Omega(\mu)$ (see Remark 1), then $f \in S H_{k, \alpha, \beta, \lambda}(\mu)$ if and only if

$$
\frac{D_{\beta, \lambda}^{k+1, \alpha} f(z)}{D_{\beta, \lambda}^{k, \alpha} f(z)} \prec p_{\mu}(z), z \in U,
$$


such that $p_{\mu}(z)=(1+2 \mu) \sqrt{\frac{1+b z}{1-z}}-2 \mu$, where $b=\frac{1+4 \mu-4 \mu^{2}}{(1+2 \mu)^{2}}$ and the branch of the square root $\sqrt{w}$ is chosen so that $\operatorname{Im} \sqrt{w} \geq 0$.

We can observe that

$$
S H_{k, 0,1,1}(\mu) \equiv S H_{k}(\mu)
$$

where the class $S H_{k}(\mu)$ was introduced in [1]. Also we have

$$
S H_{0,0,1,1}(\mu) \equiv S H(\mu),
$$

where the class $S H(\mu)$ was proposed in [15].

Definition 6. Let $0 \leq \alpha<1, \beta \geq 0, \lambda \geq 0$, and $f \in \mathcal{A}, v \in[0, \infty)$. We define the class $(v, k)_{\alpha, \beta, \lambda}-\S$ by requiring that $f \in \mathcal{S}$ and

$$
\Re\left(\frac{D_{\beta, \lambda}^{k, \alpha} f(z)}{f(z)}\right)>v\left|\frac{D_{\beta, \lambda}^{k, \alpha} f(z)}{f(z)}-1\right|,(z \in U) .
$$

Note that

$$
(v, k)_{0,1,1}-8 \equiv(v, k)-8,
$$

where the class $(v, k)-\S$ is defined and studied in [2]. if

Remark 4. (Geometric interpretation): A function $f \in(\nu, k)_{\alpha, \beta, \lambda}-\S$ if and only

$$
\frac{D_{\beta, \lambda}^{k, \alpha} f(z)}{f(z)} \prec p_{v}(z),(v \in[0, \infty), z \in U)
$$

where $p_{v}$ denoted the function which maps the unit disk conformally onto the region $\Omega_{v}$, such that $1 \in \Omega_{v}$ and

$$
\partial \Omega_{v}=\left\{u+i v: u^{2}=v^{2}(u-1)^{2}+v^{2} v^{2}\right\} .
$$

The domain $\Omega_{v}$ is elliptic for $v>1$, is hyperbolic when $0<v<1$, parabolic for $v=1$, and is the right half-plane when $v=0$.

Definition 7. Let $0 \leq \alpha<1, \beta \geq 0, \lambda \geq 0$, and $f \in \mathcal{A}, v \in[0, \infty)$. We define the class $(\nu, k)_{\alpha, \beta, \lambda}-\mathcal{C}$ with respect to the function $g \in(\nu, k)_{\alpha, \beta, \lambda}-\delta$

$$
\Re\left(\frac{D_{\beta, \lambda}^{k, \alpha} f(z)}{g(z)}\right)>v\left|\frac{D_{\beta, \lambda}^{k, \alpha} f(z)}{g(z)}-1\right|,(z \in U) .
$$

Note that

$$
(v, k)_{0,1,1}-e r \equiv(v, k)-e r,
$$

where the class $(v, k)-e \mathcal{C}$ is defined and studied in [1]. 
Remark 5. (Geometric interpretation): A function $f \in(\nu, k)_{\alpha, \beta, \lambda}-\mathcal{e}$ with respect to $g \in(\nu, k)_{\alpha, \beta, \lambda}-\S$ if and only if

$$
\frac{D_{\beta, \lambda}^{k, \alpha} f(z)}{g(z)} \prec p_{v}(z),(v \in[0, \infty), z \in U)
$$

where $p_{v}$ is defined in Remark 4. Or $\frac{D_{\beta, \lambda}^{k, \alpha} f(z)}{g(z)}$ take all values in the domain $\Omega_{\nu}$.

We need the following preliminaries in the sequel. The Libera-Pascu integral operator $L_{a}: \mathcal{A} \rightarrow \mathcal{A}$ is defined by

$$
f(z):=L_{a} F(z)=\frac{1+a}{z^{a}} \int_{0}^{z} F(t) t^{a-1} d t, a \in \mathbb{C}, \Re(a) \geq 0 .
$$

For $a=1$ we obtain the Libera integral operator, for $a=0$ we obtain the Alexander integral operator and in the cases $a=1,2,3, \ldots$ we obtain the Bernardi integral operator.

Lemma 1. ( see [7]) Let $\phi$ be convex in $U$ with $\Re\{\kappa \phi(z)+v\}>0$ for $\kappa, v \in \mathbb{C}$, also let $p(z) \in \mathscr{H}(U)$ with $p(0)=\phi(0)$ and assume that the Briot-Bouquet differential subordination

is satisfied. These imply that

$$
p(z)+\frac{z p^{\prime}(z)}{\kappa p(z)+v} \prec \phi(z), z \in U
$$

$$
p(z) \prec \phi(z), z \in U .
$$

Lemma 2. ( see [7]) Let $q$ be convex in $U$ and $\phi: U \rightarrow \mathbb{C}$ with $\Re\{\phi(z)\}>0$. If $p(z) \in \mathscr{H}(U)$ and assume that the subordination

$$
p(z)+\phi(z) \cdot z p^{\prime}(z) \prec q(z), z \in U
$$

is satisfied, then

$$
p(z) \prec q(z), z \in U .
$$

\section{THE CLASS $S H_{k, \alpha, \beta, \lambda}(\mu)$}

In this section we study the inclusion property of functions in the class $S H_{k, \alpha, \beta, \lambda}(\mu)$. Further we show that if $F \in S H_{k, \alpha, \beta, \lambda}(\mu)$ implies that for the Libera integral operator $L_{a} F(z) \in S H_{k, \alpha, \beta, \lambda}(\mu)$.

Theorem 1. Let $\mu>0,0 \leq \alpha<1, \beta \geq 1, \lambda>0$. We have

$$
S H_{k+1, \alpha, \beta, \lambda}(\mu) \subset S H_{k, \alpha, \beta, \lambda}(\mu) .
$$

Proof. Let $f \in S H_{k+1, \alpha, \beta, \lambda}(\mu)$. Denote

$$
p(z):=\frac{D_{\beta, \lambda}^{k+1, \alpha} f(z)}{D_{\beta, \lambda}^{k, \alpha} f(z)}, p(0)=1,(z \in U) .
$$


Then we have

$$
\frac{D_{\beta, \lambda}^{k+2, \alpha} f(z)}{D_{\beta, \lambda}^{k+1, \alpha} f(z)}=\frac{D_{\beta, \lambda}^{k+2, \alpha} f(z)}{D_{\beta, \lambda}^{k, \alpha} f(z)} \cdot \frac{D_{\beta, \lambda}^{k, \alpha} f(z)}{D_{\beta, \lambda}^{k+1, \alpha} f(z)}=\frac{1}{p(z)} \frac{D_{\beta, \lambda}^{k+2, \alpha} f(z)}{D_{\beta, \lambda}^{k, \alpha} f(z)} .
$$

Now, by using the definition of the operator (1.3), we verify

$$
\begin{aligned}
p^{2}(z)+\lambda z p^{\prime}(z) & =\left[\frac{D_{\beta, \lambda}^{k+1, \alpha} f(z)}{D_{\beta, \lambda}^{k, \alpha} f(z)}\right]^{2} \\
& +\lambda\left[\frac{D_{\beta, \lambda}^{k, \alpha} f(z) \cdot z\left(D_{\beta, \lambda}^{k+1, \alpha} f(z)\right)^{\prime}-D_{\beta, \lambda}^{k+1, \alpha} f(z) \cdot z\left(D_{\beta, \lambda}^{k, \alpha} f(z)\right)^{\prime}}{\left(D_{\beta, \lambda}^{k, \alpha} f(z)\right)^{2}}\right] \\
& =\left[\frac{D_{\beta, \lambda}^{k+1, \alpha} f(z)}{D_{\beta, \lambda}^{k, \alpha} f(z)}\right]^{2} \\
& +\left[\frac{D_{\beta, \lambda}^{k, \alpha} f(z) \cdot\left(D_{\beta, \lambda}^{k+2, \alpha} f(z)-(\beta-\lambda) D_{\beta, \lambda}^{k+1, \alpha} f(z)\right)-(1-\beta) z}{\left(D_{\beta, \lambda}^{k, \alpha} f(z)\right)^{2}}\right] \\
& -\left[\frac{D_{\beta, \lambda}^{k+1, \alpha} f(z) \cdot\left(D_{\beta, \lambda}^{k+1, \alpha} f(z)-(\beta-\lambda) D_{\beta, \lambda}^{k, \alpha} f(z)\right)-(1-\beta) z}{\left(D_{\beta, \lambda}^{k, \alpha} f(z)\right)^{2}}\right] \\
& =\frac{\left(D_{\beta, \lambda}^{k+1, \alpha} f(z)\right)^{2}}{\left(D_{\beta, \lambda}^{k, \alpha} f(z)\right)^{2}}+\frac{D_{\beta, \lambda}^{k, \alpha} f(z) D_{\beta, \lambda}^{k+2, \alpha} f(z)-\left(D_{\beta, \lambda}^{k+1, \alpha} f(z)\right)^{2}}{\left(D_{\beta, \lambda}^{k, \alpha} f(z)\right)^{2}} \\
& =\frac{D_{\beta, \lambda}^{k+2, \alpha} f(z)}{D_{\beta, \lambda}^{k, \alpha} f(z)} .
\end{aligned}
$$

Putting (2.2) in (2.1), we obtain

$$
\frac{D_{\beta, \lambda}^{k+2, \alpha} f(z)}{D_{\beta, \lambda}^{k+1, \alpha} f(z)}=p(z)+\lambda \frac{z p^{\prime}(z)}{p(z)},(z \in U) .
$$

Since $f \in S H_{k+1, \alpha, \beta, \lambda}(\mu)$, then in view of Remark 3, we have

$$
\frac{D_{\beta, \lambda}^{k+2, \alpha} f(z)}{D_{\beta, \lambda}^{k+1, \alpha} f(z)}=p(z)+\lambda \frac{z p^{\prime}(z)}{p(z)} \prec p_{\mu}(z),
$$

with $p(0)=p_{\mu}(0)=1, \mu>0$, and $\Re\left(p_{\mu}(z)\right)>0$. Hence, from Lemma 1 , we obtain

$$
p(z) \prec p_{\mu}(z),(z \in U),
$$


or

$$
\frac{D_{\beta, \lambda}^{k+1, \alpha} f(z)}{D_{\beta, \lambda}^{k, \alpha} f(z)} \prec p_{\mu}(z),(z \in U) .
$$

Now Remark 3 gives $f(z) \in S H_{k, \alpha, \beta, \lambda}(\mu)$.

The next results can be found in $[1,2]$ respectively.

Corollary 1. Let $\mu>0$. We have

$$
S H(\mu) \subset \& .
$$

Proof. By letting $k=0$ in Theorem 1 .

Corollary 2. Let $\mu>0$. We have

$$
S H_{n+1, \lambda}(\mu) \subset S H_{n, \lambda}(\mu) .
$$

Proof. By assuming $\alpha=0$ and $\beta=1$ in Theorem 1 .

Theorem 2. Let $\mu>0,0 \leq \alpha<1, \beta \geq 1, \lambda>0$. If $F \in S H_{k, \alpha, \beta, \lambda}(\mu)$ with $\lambda(1+$ a) $>\beta$, then the Libera-Pascu integral operator $f(z):=L_{a} F(z)$ is in $S H_{k, \alpha, \beta, \lambda}(\mu)$.

Proof. Let $F \in S H_{k, \alpha, \beta, \lambda}(\mu)$. Denote

$$
p(z):=\frac{D_{\beta, \lambda}^{k+1, \alpha} f(z)}{D_{\beta, \lambda}^{k, \alpha} f(z)}, p(0)=1,(z \in U)
$$

and assume that $0<\Re\{p(z)\} \leq 1$. From the definition of the Libera-Pascu integral operator we have

$$
(1+a) F(z)=a f(z)+z f^{\prime}(z),
$$

by using the linear operator $D_{\beta, \lambda}^{k+1, \alpha}$, we have

$$
\begin{gathered}
(1+a) D_{\beta, \lambda}^{k+1, \alpha} F(z)=a D_{\beta, \lambda}^{k+1, \alpha} f(z)+D_{\beta, \lambda}^{k+1, \alpha}\left(z f^{\prime}(z)\right) \\
=a D_{\beta, \lambda}^{k+1, \alpha} f(z)+z\left(D_{\beta, \lambda}^{k+1, \alpha} f(z)\right)^{\prime} \\
=a D_{\beta, \lambda}^{k+1, \alpha} f(z)+\frac{D_{\beta, \lambda}^{k+2, \alpha} f(z)-(\beta-\lambda) D_{\beta, \lambda}^{k+1, \alpha} f(z)-(1-\beta) z}{\lambda},
\end{gathered}
$$

or equivalently,

$$
\lambda(1+a) D_{\beta, \lambda}^{k+1, \alpha} F(z)=[\lambda(1+a)-\beta] D_{\beta, \lambda}^{k+1, \alpha} f(z)+D_{\beta, \lambda}^{k+2, \alpha} f(z)-(1-\beta) z .
$$

Similarly, we obtain

$$
\lambda(1+a) D_{\beta, \lambda}^{k, \alpha} F(z)=[\lambda(1+a)-\beta] D_{\beta, \lambda}^{k, \alpha} f(z)+D_{\beta, \lambda}^{k+1, \alpha} f(z)-(1-\beta) z .
$$


Then by using (2.4) and (2.3) we have

$$
\begin{aligned}
\frac{D_{\beta, \lambda}^{k+1, \alpha} F(z)}{D_{\beta, \lambda}^{k, \alpha} F(z)} & =\frac{[\lambda(1+a)-\beta] \frac{D_{\beta, \lambda}^{k+1, \alpha} f(z)}{D_{\beta, \lambda}^{k, \alpha} f(z)}+\frac{D_{\beta, \lambda}^{k+2, \alpha} f(z)}{D_{\beta, \lambda}^{k+1, \alpha} f(z)} \cdot \frac{D_{\beta, \lambda}^{k+1, \alpha} f(z)}{D_{\beta, \lambda}^{k, \alpha} f(z)}-\frac{(1-\beta) z}{D_{\beta, \lambda}^{k, \alpha} f(z)}}{[\lambda(1+a)-\beta]+\frac{D_{\beta, \lambda}^{k+1, \alpha} f(z)}{D_{\beta, \lambda}^{k, \alpha}}-\frac{(1-\beta) z}{D_{\beta, \lambda}^{k, \alpha} f(z)}} \\
& :=\frac{[\lambda(1+a)-\beta] p(z)+\frac{D_{\beta, \lambda}^{k+2, \alpha} f(z)}{D_{\beta, \lambda}^{k+1, \alpha} f(z)} \cdot p(z)+\varphi(z)}{[\lambda(1+a)-\beta]+p(z)+\varphi(z)} \\
& =\frac{[\lambda(1+a)-\beta] p(z)+p^{2}(z)+\lambda z p^{\prime}(z)+\varphi(z)}{[\lambda(1+a)-\beta]+p(z)+\varphi(z)} \\
& =\frac{p(z)\{[\lambda(1+a)-\beta]+p(z)+\varphi(z)\}+\lambda z p^{\prime}(z)+\varphi(z)(1-p(z))}{[\lambda(1+a)-\beta]+p(z)+\varphi(z)} \\
& =p(z)+z p^{\prime}(z) \cdot\left(\frac{\lambda+\frac{\varphi(z)(1-p(z))}{z p^{\prime}(z)}}{[\lambda(1+a)-\beta]+p(z)+\varphi(z)}\right) \\
& :=p(z)+z p^{\prime}(z) \cdot \phi(z),
\end{aligned}
$$

where $\Re(\phi(z))>0$ as $z \rightarrow 1^{+}$. From $F \in H S_{k, \alpha, \beta, \lambda}(\mu)$, we have

$$
p(z)+z p^{\prime}(z) \cdot \phi(z) \prec p_{\mu}(z),
$$

thus in view of Lemma 2, we obtain

$$
p(z) \prec p_{\mu}(z),(z \in U)
$$

or

$$
\frac{D_{\beta, \lambda}^{k+1, \alpha} f(z)}{D_{\beta, \lambda}^{k, \alpha} f(z)} \prec p_{\mu}(z), p(0)=1,(z \in U) .
$$

Implies $f \in H S_{k, \alpha, \beta, \lambda}(\mu)$. This completes the proof.

The next results can be found in [2].

Corollary 3. Let $\mu>0$. If $F \in S H_{n, \lambda}(\mu)$. Then the $f \in S H_{n, \lambda}(\mu)$ where $f$ is the Libera-Pascu integral operator.

Proof. Assume $\alpha=0$ and $\beta=1$. Hence $\phi(z)=\frac{\lambda}{[\lambda(1+a)-1]+p(z)}$ in Theorem 2.

\section{The CLASS $(\nu, k)_{\alpha, \beta, \lambda}-e e$}

In this section, we show that for any function $F \in \mathcal{A}$ in the classes $(v, k)_{\alpha, \beta, \lambda}-\delta$ and $(\nu, k)_{\alpha, \beta, \lambda}-\mathcal{C}$, the Libera-Pascu integral operator acting on $F$ is also belongs to these classes. Moreover, some well known subclasses are obtained as special cases of our results. 
Theorem 3. If $F \in(v, k)_{\alpha, \beta, \lambda}-\gamma, v \in[0, \infty)$ and $0 \leq \alpha<1, \beta>0, \lambda>0$, then Libera-Pascu integral operator $f(z)=L_{a} F(z) \in(\nu, k)_{\alpha, \beta, \lambda}-\S$.

Proof. Let $F \in(v, k)_{\alpha, \beta, \lambda}-8$. Consider

$$
p(z):=\frac{D_{\beta, \lambda}^{k, \alpha} f(z)}{f(z)}, p(0)=1,(z \in U) .
$$

From the definition of the Libera-Pascu integral operator we obtain

$$
(1+a) F(z)=a f(z)+z f^{\prime}(z)
$$

By using the linear operator $D_{\beta, \lambda}^{k, \alpha}$, we have

$$
\begin{aligned}
(1+a) D_{\beta, \lambda}^{k, \alpha} F(z) & =a D_{\beta, \lambda}^{k, \alpha} f(z)+D_{\beta, \lambda}^{k, \alpha}\left(z f^{\prime}(z)\right) \\
& =a D_{\beta, \lambda}^{k, \alpha} f(z)+z\left(D_{\beta, \lambda}^{k, \alpha} f(z)\right)^{\prime} \\
& =a D_{\beta, \lambda}^{k, \alpha} f(z)+\frac{D_{\beta, \lambda}^{k+1, \alpha} f(z)-(\beta-\lambda) D_{\beta, \lambda}^{k, \alpha} f(z)-(1-\beta) z}{\lambda} \\
& =\left[a+\frac{\lambda-\beta}{\lambda}\right] D_{\beta, \lambda}^{k, \alpha} f(z)+\frac{1}{\lambda} D_{\beta, \lambda}^{k+1, \alpha} f(z)+\frac{(\beta-1) z}{\lambda} \\
& :=A D_{\beta, \lambda}^{k, \alpha} f(z)+B D_{\beta, \lambda}^{k+1, \alpha} f(z)+\varphi(z) .
\end{aligned}
$$

From (3.2) and (3.3) we obtain

$$
\begin{aligned}
\frac{D_{\beta, \lambda}^{k, \alpha} F(z)}{F(z)} & =\frac{A D_{\beta, \lambda}^{k, \alpha} f(z)+B D_{\beta, \lambda}^{k+1, \alpha} f(z)+\varphi(z)}{a f(z)+z f^{\prime}(z)} \\
& =\frac{f(z)\left[A \frac{D_{\beta, \lambda}^{k, \alpha} f(z)}{f(z)}+B \frac{D_{\beta, \lambda}^{k+1, \alpha} f(z)}{f(z)}+\frac{\varphi(z)}{f(z)}\right]}{f(z)\left[a+\frac{z f^{\prime}(z)}{f(z)}\right]} \\
& =\frac{A \frac{D_{\beta, \lambda}^{k, \alpha} f(z)}{f(z)}+B \frac{D_{\beta, \lambda}^{k+1, \alpha} f(z)}{f(z)}+\frac{\varphi(z)}{f(z)}}{a+\frac{z f^{\prime}(z)}{f(z)}}
\end{aligned}
$$

From (3.1) we have 


$$
\begin{aligned}
z p^{\prime}(z) & =z \frac{f(z)\left[D_{\beta, \lambda}^{k, \alpha} f(z)\right]^{\prime}-D_{\beta, \lambda}^{k, \alpha} f(z) \cdot f^{\prime}(z)}{f^{2}(z)} \\
& =\frac{z\left[D_{\beta, \lambda}^{k, \alpha} f(z)\right]^{\prime}}{f(z)}-\frac{D_{\beta, \lambda}^{k, \alpha}}{f(z)} \cdot \frac{z f^{\prime}(z)}{f(z)} \\
& =\frac{\frac{D_{\beta, \lambda}^{k+1, \alpha} f(z)-(\beta-\lambda) D_{\beta, \lambda}^{k, \alpha} f(z)-(1-\beta) z}{\lambda}}{f(z)}-\frac{D_{\beta, \lambda}^{k, \alpha} f(z)}{f(z)} \cdot \frac{z f^{\prime}(z)}{f(z)} \\
& :=\frac{B D_{\beta, \lambda}^{k+1, \alpha} f(z)}{f(z)}-\frac{C D_{\alpha, \beta, \lambda}^{\delta} f(z)}{f(z)}+\frac{\varphi(z)}{f(z)}-p(z) \cdot \frac{z f^{\prime}(z)}{f(z)} \\
& =\frac{B D_{\beta, \lambda}^{k+1, \alpha} f(z)}{f(z)}-C p(z)+\frac{\varphi(z)}{f(z)}-p(z) \cdot \frac{z f^{\prime}(z)}{f(z)},
\end{aligned}
$$

where $C:=\frac{(\beta-\lambda)}{\lambda}$. Hence

$$
\frac{B D_{\beta, \lambda}^{k+1, \alpha} f(z)}{f(z)}=z p^{\prime}(z)+p(z)\left[C+\frac{z f^{\prime}(z)}{f(z)}\right]-\frac{\varphi(z)}{f(z)} .
$$

Substitute (3.6) in (3.4), then we obtain

$$
\begin{aligned}
\frac{D_{\beta, \lambda}^{k, \alpha} F(z)}{F(z)} & =\frac{A p(z)+B \frac{D_{\beta, \lambda}^{k+1, \alpha} f(z)}{f(z)}+\frac{\varphi(z)}{f(z)}}{a+\frac{z f^{\prime}(z)}{f(z)}} \\
& =\frac{z p^{\prime}(z)+p(z)\left[A+C+\frac{z f^{\prime}(z)}{f(z)}\right]}{a+\frac{z f^{\prime}(z)}{f(z)}} \\
& =\frac{z p^{\prime}(z)+p(z)\left[a+\frac{z f^{\prime}(z)}{f(z)}\right]}{a+\frac{z f^{\prime}(z)}{f(z)}} \\
& =p(z)+\frac{1}{a+\frac{z f^{\prime}(z)}{f(z)}} z p^{\prime}(z) .
\end{aligned}
$$

From the hypothesis, we have

$$
\frac{D_{\beta, \lambda}^{k, \alpha} F(z)}{F(z)} \prec p_{v}(z),(v \in[0, \infty), z \in U) .
$$

Consequently, we have

$$
p(z)+\frac{1}{a+\frac{z f^{\prime}(z)}{f(z)}} z p^{\prime}(z) \prec p_{v}(z),(v \in[0, \infty), z \in U) .
$$


Now in view of Lemma 2, we get

$$
p(z) \prec p_{v}(z),(v \in[0, \infty), z \in U) .
$$

Equivalently

$$
\frac{D_{\beta, \lambda}^{k, \alpha} f(z)}{f(z)} \prec p_{v}(z), \quad(v \in[0, \infty), z \in U),
$$

which implies $f(z)=L_{a} F(z) \in(\nu, k)_{\alpha, \beta, \lambda}-\S$.

The next result can be found in [2].

Corollary 4. If $F \in(v, n)-\S, v \in[0, \infty)$, then Libera-Pascu integral operator $f(z)=L_{a} F(z) \in(\nu, n)-s$.

Proof. Let $\alpha=0, \beta=\lambda=1$ in Theorem 3.

Corollary 5. If $F \in(v, k)_{\lambda}-\S, v \in[0, \infty)$, then Libera-Pascu integral operator $f(z)=L_{a} F(z) \in(v, k)_{\lambda}-\varsigma$.

Proof. Put $\alpha=0, \beta=1$ in Theorem 3. Note that in this case we have Al-Oboudi's differential operator.

Theorem 4. If $F \in(v, k)_{\alpha, \beta, \lambda}-\mathcal{C}, v \in[0, \infty)$ and $0 \leq \alpha<1, \beta>0, \lambda>0$, with respect to the function $G(z) \in(v, k)_{\alpha, \beta, \lambda}-\S$, then the Libera-Pascu integral operator $f(z)=L_{a} F(z) \in(v, k)_{\alpha, \beta, \lambda}-\mathcal{C}^{\mathcal{C}}$ with respect to the function $g(z)=$ $L_{a} G(z) \in(\nu, k)_{\alpha, \beta, \lambda}-8$.

Proof. Let $F \in(\nu, k)_{\alpha, \beta, \lambda}-\mathcal{e}$ and

$$
p(z):=\frac{D_{\beta, \lambda}^{k, \alpha} f(z)}{g(z)}, p(0)=1,(z \in U) .
$$

Differentiating the Libera-Pascu integral operator $f(z)=L_{a} F(z)$ and operating by $D_{\beta, \lambda}^{k, \alpha}$, we have

$$
(1+a) D_{\beta, \lambda}^{k, \alpha} F(z)=A D_{\beta, \lambda}^{k, \alpha} f(z)+B D_{\beta, \lambda}^{k+1, \alpha} f(z)+\varphi(z),
$$

where $A, B$ and $\varphi(z)$ are defined in Theorem 3. We also observe that

$$
(1+a) G(z)=a g(z)+z g^{\prime}(z) .
$$


By using (3.9) and (3.10) we get

$$
\begin{aligned}
\frac{D_{\beta, \lambda}^{k, \alpha} F(z)}{G(z)} & =\frac{A D_{\beta, \lambda}^{k, \alpha} f(z)+B D_{\beta, \lambda}^{k+1, \alpha} f(z)+\varphi(z)}{a g(z)+z g^{\prime}(z)} \\
& =\frac{g(z)\left[A \frac{D_{\beta, \lambda}^{k, \alpha} f(z)}{g(z)}+B \frac{D_{\beta, \lambda}^{k+1, \alpha} f(z)}{g(z)}+\frac{\varphi(z)}{g(z)}\right]}{g(z)\left[a+\frac{z g^{\prime}(z)}{g(z)}\right]} \\
& =\frac{A \frac{D_{\beta, \lambda}^{k, \alpha} f(z)}{g(z)}+B \frac{D_{\beta, \lambda}^{k+1, \alpha} f(z)}{g(z)}+\frac{\varphi(z)}{g(z)}}{a+\frac{z g^{\prime}(z)}{g(z)}}
\end{aligned}
$$

From (3.8) we have

$$
\begin{aligned}
z p^{\prime}(z) & =z \frac{g(z)\left[D_{\beta, \lambda}^{k, \alpha} f(z)\right]^{\prime}-D_{\beta, \lambda}^{k, \alpha} f(z) \cdot g^{\prime}(z)}{g^{2}(z)} \\
& =\frac{z\left[D_{\beta, \lambda}^{k, \alpha} f(z)\right]^{\prime}}{g(z)}-\frac{D_{\beta, \lambda}^{k, \alpha} f(z)}{g(z)} \cdot \frac{z g^{\prime}(z)}{g(z)} \\
& =\frac{D_{\beta, \lambda}^{k+1, \alpha} f(z)-(\beta-\lambda) D_{\beta, \lambda}^{k, \alpha} f(z)-(1-\beta) z}{\lambda g(z)}-\frac{D_{\beta, \lambda}^{k, \alpha} f(z)}{g(z)} \cdot \frac{z g^{\prime}(z)}{g(z)} \\
& :=\frac{B D_{\beta, \lambda}^{k+1, \alpha} f(z)}{g(z)}-\frac{C D_{\beta, \lambda}^{k, \alpha} f(z)}{g(z)}+\frac{\varphi(z)}{g(z)}-p(z) \cdot \frac{z g^{\prime}(z)}{g(z)} \\
& =\frac{B D_{\beta, \lambda}^{k+1, \alpha} f(z)}{g(z)}-C p(z)-p(z) \cdot \frac{z g^{\prime}(z)}{g(z)}+\frac{\varphi(z)}{g(z)},
\end{aligned}
$$

where $C$ defined in Theorem 3. Hence

$$
\frac{B D_{\beta, \lambda}^{k+1, \alpha} f(z)}{g(z)}=z p^{\prime}(z)+p(z)\left[C+\frac{z g^{\prime}(z)}{g(z)}\right]-\frac{\varphi(z)}{g(z)} .
$$

Substitute (3.13) in (3.11) we obtain

$$
\begin{aligned}
\frac{D_{\beta, \lambda}^{k, \alpha} F(z)}{G(z)} & =p(z)+\frac{1}{a+\frac{z g^{\prime}(z)}{g(z)}} z p^{\prime}(z) \\
& :=p(z)+\phi(z) z p^{\prime}(z),
\end{aligned}
$$

where $\Re\{\phi\}>0, z \in U$. Thus in view of Lemma 2, we obtain

$$
p(z) \prec p_{v}(z),(v \in[0, \infty), z \in U)
$$


or equivalently

$$
\frac{D_{\beta, \lambda}^{k, \alpha} f(z)}{g(z)} \prec p_{v}(z), \quad(v \in[0, \infty), z \in U) .
$$

Hence $f(z)=L_{a} F(z) \in(\nu, k)_{\alpha, \beta, \lambda}-\bigodot \mathcal{C}$ with respect to the function $g(z)=L_{a} G(z) \in$ $(v, k)_{\alpha, \beta, \lambda}-\S$.

The next result can be found in [2].

Corollary 6. If $F \in(v, k)-\mathcal{e}, v \in[0, \infty)$ with respect to the function $G(z) \in$ $(v, k)-8$ then Libera-Pascu integral operator $f(z)=L_{a} F(z) \in(v, n)-$ Ce with respect to the function $g(z)=L_{a} G(z) \in(v, k)-\S$.

Proof. Let $\alpha=0, \beta=\lambda=1$ in Theorem 4.

Corollary 7. If $F \in(v, k)_{\lambda}-\mathcal{e} \mathcal{C}, v \in[0, \infty)$ and $\lambda>0$, with respect to the function $G(z) \in(v, k)_{\lambda}-8$, then Libera-Pascu integral operator $f(z)=L_{a} F(z) \in$ $(v, k)_{\lambda}$-ee with respect to the function $g(z)=L_{a} G(z) \in(\nu, k)_{\lambda}-8$.

Proof. Put $\alpha=0, \beta=1$ in Theorem 4. Note that in this case we have Al-Oboudi's differential operator.

\section{THE CLASS $(\nu, k)_{\alpha, \beta, \lambda}-\S$}

In this section, we determine coefficient bounds for functions of the form (1.1), with positive and negative coefficients, in the class $(\nu, k)_{\alpha, \beta, \lambda}-8$. This study is required in the next section.

Theorem 5. A sufficient condition for a function $f(z)$ of the form (1.1) to be in $\operatorname{class}(v, k)_{\alpha, \beta, \lambda}-8$ is

$$
\sum_{n=2}^{\infty}\left|a_{n}\right|\left[\left(\Psi_{n, k}(\alpha, \beta, \lambda)-1\right)(1+v)+1\right]<1,
$$

where $v \in[0, \infty)$ and $0 \leq \alpha<1, \beta>0, \lambda>0$.

Proof. It suffices to show that

$$
v\left|\frac{D_{\beta, \lambda}^{k, \alpha} f(z)}{f(z)}-1\right|-\Re\left(\frac{D_{\beta, \lambda}^{k, \alpha} f(z)}{f(z)}-1\right) \leq 1 .
$$

We obtain

$$
\begin{gathered}
v\left|\frac{D_{\beta, \lambda}^{k, \alpha} f(z)}{f(z)}-1\right|-\Re\left(\frac{D_{\beta, \lambda}^{k, \alpha} f(z)}{f(z)}-1\right) \leq(1+v)\left|\frac{D_{\beta, \lambda}^{k, \alpha} f(z)}{f(z)}-1\right| \\
\leq \frac{(1+v) \sum_{n=2}^{\infty}\left|a_{n}\right|\left(\Psi_{n, k}(\alpha, \beta, \lambda)-1\right)|z|^{n-1}}{1-\sum_{n=2}^{\infty}\left|a_{n}\right||z|^{n-1}}
\end{gathered}
$$




$$
\leq \frac{(1+v) \sum_{n=2}^{\infty}\left|a_{n}\right|\left(\Psi_{n, k}(\alpha, \beta, \lambda)-1\right)}{1-\sum_{n=2}^{\infty}\left|a_{n}\right|} .
$$

This last expression is bounded above by 1 if

$$
\sum_{n=2}^{\infty}\left|a_{n}\right|\left[\left(\Psi_{n, k}(\alpha, \beta, \lambda)-1\right)(1+v)+1\right]<1,
$$

and the proof is complete.

Now we prove that the condition (4.1) is also necessary for $f$ with negative coefficients. Let $\mathcal{T}$ be the class of all analytic functions of the form

$$
f(z)=z-\sum_{n=2}^{\infty} a_{n} z^{n},\left(a_{n} \geq 0, z \in U\right) .
$$

Then we have the following result.

Theorem 6. A sufficient and necessary condition for $f \in \mathcal{T}$ to be in the class $(v, k)_{\alpha, \beta, \lambda}-\mathcal{T} \delta=(v, k)_{\alpha, \beta, \lambda}-\delta \cap \mathcal{T}$ (here $v \in[0, \infty)$ and $\left.0 \leq \alpha<1, \beta>0, \lambda>0\right)$, is that

$$
\sum_{n=2}^{\infty} a_{n}\left[\left(\Psi_{n, k}(\alpha, \beta, \lambda)-1\right)(1+v)+1\right]<1 .
$$

Proof. In view of Theorem 1, we need to prove only the necessity. If $f \in(\nu, k)_{\alpha, \beta, \lambda}-$ $\tau \&$ and $z$ is real, then

$$
\begin{aligned}
1 & >v\left|\frac{D_{\beta, \lambda}^{k, \alpha} f(z)}{f(z)}-1\right|-\Re\left(\frac{D_{\beta, \lambda}^{k, \alpha} f(z)}{f(z)}-1\right) \\
& \geq-(1+v) \Re\left(\frac{D_{\beta, \lambda}^{k, \alpha} f(z)}{f(z)}-1\right) \\
& =-(1+v) \Re\left(\frac{-\sum_{n=2}^{\infty} a_{n}\left(\Psi_{n, k}(\alpha, \beta, \lambda)-1\right) z^{n-1}}{1-\sum_{n=2}^{\infty} a_{n} z^{n-1}}\right) .
\end{aligned}
$$

Letting $z \rightarrow 1$ along the real axis, we obtain the desired inequality

$$
\sum_{n=2}^{\infty} a_{n}\left[\left(\Psi_{n, k}(\alpha, \beta, \lambda)-1\right)(1+v)+1\right]<1 .
$$

Corollary 8. The extreme points of $(\nu, k)_{\alpha, \beta, \lambda}-\mathcal{T} \&$ are the functions given by

$$
f_{1}(z)=1 \text {, and } f_{n}(z)=z-\frac{1}{\left[\left(\Psi_{n, k}(\alpha, \beta, \lambda)-1\right)(1+v)+1\right]} z^{n},
$$

where $n=2,3, \ldots, v \in[0, \infty)$ and $0 \leq \alpha<1, \beta>0, \lambda>0$. 


\section{Ceś́́ro sum}

Recently, Silverman [14] determined sharp lower bounds on the real part of the quotients between the normalized starlike or convex functions and their sequences of partial sums:

$$
\Re\left\{\frac{f(z)}{f_{N}(z)}\right\}, \Re\left\{\frac{f_{N}(z)}{f(z)}\right\}, \Re\left\{\frac{f^{\prime}(z)}{f_{N}^{\prime}(z)}\right\} \text {, and } \Re\left\{\frac{f_{N}^{\prime}(z)}{f^{\prime}(z)}\right\} .
$$

In this section, we consider the Cesáro sum $\sigma_{m}$ of functions in the class $(\nu, k)_{\alpha, \beta, \lambda}-$ $\delta$ and obtain sharp lower bounds for the ratios of real part of $f(z)$ and $\sigma_{m}$, and also of $f^{\prime}(z)$ and $\sigma_{m}^{\prime}$. Let us first construct the Cesáro sum $\sigma_{m}(z)$ of functions $f \in \mathcal{A}$ by

$$
\begin{aligned}
\sigma_{m}(z) & =\frac{1}{m+1} \sum_{n=0}^{m} s_{n}(z) \\
& =\frac{1}{m+1}\left[s_{0}(z)+s_{1}(z)+. .+s_{m}(z)\right] \\
& =\frac{1}{m+1}\left[a_{0}+\left(a_{0}+a_{1} z\right)+. .+\left(a_{0}+. .+a_{m} z^{m}\right)\right] \\
& =\frac{1}{m+1}\left[(m+1) a_{0}+m a_{1} z+. .+a_{m} z^{m}\right] \\
& =\sum_{n=0}^{m}\left(\frac{m-n+1}{m+1}\right) a_{m} z^{n} \\
& =f(z) * \sum_{n=0}^{m}\left(\frac{m-n+1}{m+1}\right) z^{n} \\
& :=f(z) * g_{m}(z)
\end{aligned}
$$

where $s_{0}(z)=0, s_{1}(z)=z$. Note that the classical Cesáro means play an important role in geometric function theory (see $[6,12,13])$. In the sequel, we will make use of the result that $\Re\left\{\frac{(1+w(z))}{(1-w(z))}\right\}>0, \quad(z \in U)$ if and only if $w(z)=\sum_{n=1}^{\infty} c_{n} z^{n}$ satisfies the inequality $|w(z)|<|z|$.

Theorem 7. Let the function $f(z)$ of the form (1.1) satisfies condition (4.1), then

$$
\Re\left\{\frac{f(z)}{\sigma_{m}(z)}\right\} \geq \frac{\left(\Psi_{m+1, k}(\alpha, \beta, \lambda)-1\right)(1+v)}{\left(\Psi_{m+1, k}(\alpha, \beta, \lambda)-1\right)(1+v)+1},(z \in U),
$$

where $\sigma_{m}(z)$ defined in (5.1).

Proof. Assume that $f \in \mathcal{A}$ and satisfies (4.1). By setting

$$
d_{m}:=\left[\left(\Psi_{m, k}(\alpha, \beta, \lambda)-1\right)(1+v)+1\right], \quad C(n, m):=\frac{m-n+1}{m+1}
$$


and

$$
\begin{aligned}
w(z) & =d_{m+1}\left\{\frac{f(z)}{\sigma_{m}(z)}-\left(1-\frac{1}{d_{m+1}}\right)\right\} \\
& =1+\frac{d_{m+1}\left[\sum_{n=2}^{\infty} a_{n} z^{n-1}-\sum_{n=2}^{m} C(n, m) a_{n} z^{n-1}\right]}{1+\sum_{n=2}^{m} C(n, m) a_{n} z^{n-1}}
\end{aligned}
$$

when $C(n, m) \rightarrow 1$, we find that

$$
\begin{aligned}
\left|\frac{w(z)-1}{w(z)+1}\right| & \leq \frac{d_{m+1} \sum_{n=m+1}^{\infty}\left|a_{n}\right|}{2-2 \sum_{n=2}^{m}\left|a_{n}\right|-d_{m+1} \sum_{n=m+1}^{\infty}\left|a_{n}\right|} \\
& \leq 1, \quad(z \in U)
\end{aligned}
$$

if and only if

$$
2 d_{m+1} \sum_{n=m+1}^{\infty}\left|a_{n}\right| \leq 2-2 \sum_{n=2}^{m}\left|a_{n}\right|
$$

which is equivalent to

$$
\sum_{n=2}^{m}\left|a_{n}\right|+d_{m+1} \sum_{n=m+1}^{\infty}\left|a_{n}\right| \leq 1 .
$$

In order to see that

$$
f(z)=z+\frac{z^{m+1}}{d_{m+1}}, \quad(z \in U)
$$

gives sharp result, we observe that for $z=r e^{\frac{\pi i}{m}}$

$$
\frac{f(z)}{\sigma_{m}(z)}=1+\frac{z^{m}}{d_{m+1}} \rightarrow 1-\frac{1}{d_{m+1}} \text { as } z \rightarrow 1^{-} .
$$

Hence the proof is complete.

In the same manner of Theorem 7, we can verify the following result

Theorem 8. Let the function $f(z)$ of the form (1.1) satisfies condition (4.1), then

$$
\Re\left\{\frac{f^{\prime}(z)}{\sigma_{m}^{\prime}(z)}\right\} \geq \frac{\left(\Psi_{m+1, k}(\alpha, \beta, \lambda)-1\right)(1+v)-m}{\left(\Psi_{m+1, k}(\alpha, \beta, \lambda)-1\right)(1+v)+1},(z \in U),
$$

where $\sigma_{m}(z)$ defined in (5.1).

Proof. Assume that $f \in \mathcal{A}$ and satisfies (4.1). By letting

$$
\begin{aligned}
w(z) & =d_{m+1}\left\{\frac{f^{\prime}(z)}{\sigma_{m}^{\prime}(z)}-\left(1-\frac{m+1}{d_{m+1}}\right)\right\} \\
& =1+\frac{\frac{m+1}{d_{m+1}}\left[\sum_{n=2}^{\infty} n a_{n} z^{n-1}-\sum_{n=2}^{m} C(n, m) n a_{n} z^{n-1}\right]}{1+\sum_{n=2}^{m} C(n, m) n a_{n} z^{n-1}},
\end{aligned}
$$

where $d_{m}$ is defined in Theorem 7 . 


\section{REFERENCES}

[1] M. Acu, "On a subclass of $n$-starlike functions associated with some hyperbola," Gen. Math., vol. 13, no. 1, pp. 91-98, 2005.

[2] M. Acu, "On a subclass of $n$-uniformly close to convex functions," Gen. Math., vol. 14, no. 1, pp. 55-64, 2006.

[3] F. M. Al-Oboudi, "On univalent functions defined by a generalized Sălăgean operator," Int. J. Math. Math. Sci., no. 25-28, pp. 1429-1436, 2004.

[4] F. M. Al-Oboudi and K. A. Al-Amoudi, "On classes of analytic functions related to conic domains," J. Math. Anal. Appl., vol. 339, no. 1, pp. 655-667, 2008.

[5] B. C. Carlson and D. B. Shaffer, "Starlike and prestarlike hypergeometric functions," SIAM J. Math. Anal., vol. 15, no. 4, pp. 737-745, 1984.

[6] M. Darus and R. W. Ibrahim, "On Cesáro means for Fox-Wright functions,” J. Math. Stat., vol. 4, no. 3, pp. 156-160, 2008.

[7] S. S. Miller and P. T. Mocanu, "Differential subordinations and univalent functions," Michigan Math. J., vol. 28, no. 2, pp. 157-172, 1981.

[8] S. S. Miller and P. T. Mocanu, Differential subordinations. Theory and applications, ser. Monographs and Textbooks in Pure and Applied Mathematics. New York: Marcel Dekker, Inc., 2000, vol. 225.

[9] S. S. Miller and P. T. Mocanu, "Subordinants of differential superordinations," Complex Var. Theory Appl., vol. 48, no. 10, pp. 815-826, 2003.

[10] S. Owa, "On the distortion theorems. I," Kyungpook Math. J., vol. 18, no. 1, pp. 53-59, 1978.

[11] S. Owa and H. M. Srivastava, "Univalent and starlike generalized hypergeometric functions," Canad. J. Math., vol. 39, no. 5, pp. 1057-1077, 1987.

[12] S. Ruscheweyh, "Geometric properties of the Cesàro means," Results Math., vol. 22, no. 3-4, pp. 739-748, 1992.

[13] S. Ruscheweyh and L. C. Salinas, "Subordination by Cesàro means," Complex Variables Theory Appl., vol. 21, no. 3-4, pp. 279-285, 1993.

[14] H. Silverman, "Partial sums of starlike and convex functions," J. Math. Anal. Appl., vol. 209, no. 1, pp. 221-227, 1997.

[15] J. Stankiewicz and A. Wiśniowska, "Starlike functions associated with some hyperbola," Zeszyty Nauk. Politech. Rzeszowskiej Mat., no. 19, pp. 117-126, 1996.

[16] G. c. Sălăgean, "Subclasses of univalent functions," in Complex analysis-fifth Romanian-Finnish seminar, Part 1(Bucharest, 1981), ser. Lecture Notes in Math., vol. 1013. Berlin: Springer, 1983, pp. 362-372.

Authors' addresses

Rabha W. Ibrahim

University Malaya, Institute of Mathematical Sciences, Kuala Lumpur, 50603, Malaysia

E-mail address: rabhaibrahimeyahoo.com

\section{Darus}

Universiti Kebangsaan Malaysia, School of Mathematical Sciences, Bangi 43600, Malaysia

E-mail address: maslina@ukm.my 